\title{
A SIMPLE AND EFFECTIVE DISPLAY FOR NIGHT VISION SYSTEMS
}

\author{
Omer Tsimhoni, Michael J. Flannagan, Mary Lynn Mefford, and Naoko Takenobu \\ University of Michigan Transportation Research Institute \\ Ann Arbor, MI, U.S.A. \\ E-mail: omert@umich.edu
}

\begin{abstract}
Summary: The next generation of automotive night vision systems will likely continue to display to the driver enhanced images of the forward driving scene. In some displays there may also be highlighting of pedestrians and animals, which has been argued to be the primary safety goal of night vision systems. We present here the method that was used to design a conceptual display for night vision systems. Although the primary focus of the method is on safety analysis, consideration is given to driver performance with the system, and exposure to alerts. It also addresses user acceptance and annoyance, distraction, and expected behavior adaptation. The resulting driver interface is a simple and potentially effective display for night vision systems. It consists of a pedestrian icon that indicates when there are pedestrians near the future path of the vehicle. An initial prototype of this night-vision DVI was tested on the road and showed promising results despite its simplicity. It improved pedestrian detection distance from 34 to $44 \mathrm{~m}$ and decreased the overall ratio of missed pedestrians from $13 \%$ to $5 \%$, correspondingly. The improvement may be attributable to the icon alerting the driver to the presence of a pedestrian. In this experiment, the drivers were probably more alert to the possible presence of pedestrians than drivers in the real world, suggesting that the effect of the icon might be even larger in actual use.
\end{abstract}

\section{BACKGROUND}

One of the most critical features of a driver assistance or safety system is the driver-vehicle interface (DVI). The DVI must be designed so that it can get the driver's attention and evoke an effective response under the time pressure of an emergency situation. The difficulty of achieving that is heightened by the fact that emergency situations are rare, so that there is little chance for the driver to learn the characteristics of the system. The rarity of real emergency situations also means that even a low rate of false alarms can undermine the driver's faith in the system, and possibly create distraction if the system's signaling is made strong enough to get the attention of a driver who is not paying attention. In this study, we describe and partially test the potential effectiveness of a very simple interface for a night vision system. The display involved is much simpler than the video displays that are currently used in most night vision systems, and the information that it provides to the driver is correspondingly limited. However, the proposed system is intended to address the main safety problem that has been attributed to darknessspecifically, pedestrian crashes. Furthermore, the display involved may represent a particularly desirable balance by being nonintrusive enough to reduce problems with false alarms and distractions, while still being salient enough to evoke an effective response from a driver under the nighttime conditions for which it is designed. 


\section{METHOD}

The method that we used for the design of a driver-vehicle interface for night vision systems consisted of several analysis components: safety analysis, driver performance with the system, exposure to alerts, user acceptance and annoyance, distraction, and expected behavior adaptation.

Safety analysis. The primary focus of the method is on safety analysis of the situations that the systems should address. The underlying notion is that the ability of new technology to provide information to the driver is not sufficient to justify the driver's need for that information. In the case of night vision systems, it is possible to show the driver an enhanced video image of the road scene ahead, but it is not clear that such information would prevent crashes. Crash data suggest that the main potential safety benefit of automotive night vision systems is in assisting drivers to detect and avoid hitting pedestrians, animals, and cyclists (Rumar, 2003). To achieve this potential safety benefit, a successful implementation of a night vision system should only focus on pedestrians, animals, and cyclists. Sullivan and Flannagan (2001) estimated that the nighttime deaths of about 2,300 pedestrians in the U.S. each year can be attributed to darkness. Night vision systems could be cost effective and valuable to society if they prevented some of those deaths. It is noted that the probability of any particular driver being involved in a pedestrian crash is very low. For most drivers, it is less than once in their lifetime. An estimation of vehicle miles travelled and driver years between crashes and injuries is shown in Table 1.

Table 1. Vehicle-pedestrian and vehicle-animal crash estimates

\begin{tabular}{|l|l|l|l|}
\hline \multicolumn{1}{|c|}{ Description } & \multicolumn{1}{c|}{ Estimate } & \multicolumn{1}{c|}{ Source } \\
\hline \hline $\begin{array}{l}\text { Number of pedestrians killed in the U.S. } \\
\text { annually due to darkness }\end{array}$ & 2,300 deaths & $\begin{array}{l}\text { Sullivan and Flannagan, } \\
2001\end{array}$ \\
\hline $\begin{array}{l}\text { Frequency of a driver experiencing a fatal } \\
\text { pedestrian crash attributed to darkness }\end{array}$ & $\begin{array}{l}1 \text { per } 322 \text { million } \\
\text { vehicle miles traveled }\end{array}$ & $\begin{array}{l}1 \text { in } 85,000 \text { driver } \\
\text { years }\end{array}$ & $\begin{array}{l}\text { Estimated based on } \\
\text { NHTSA, 2005 }\end{array}$ \\
\hline $\begin{array}{l}\text { Frequency of a driver experiencing an injury } \\
\text { pedestrian crash attributed to darkness }\end{array}$ & $\begin{array}{l}1 \text { per } 26.5 \text { million } \\
\text { vehicle miles traveled }\end{array}$ & $\begin{array}{l}1 \text { in 7,500 driver } \\
\text { years }\end{array}$ & $\begin{array}{l}\text { Estimated based on } \\
\text { NHTSA, 2005 }\end{array}$ \\
\hline $\begin{array}{l}\text { Frequency of a driver in the upper Midwest } \\
\text { U.S. being involved in a deer crash }\end{array}$ & $\begin{array}{l}1 \text { per } 2.5 \text { million } \\
\text { vehicle miles traveled }\end{array}$ & 1 in 166 driver years & Knapp et al., 2005 \\
\hline
\end{tabular}

Driver performance with the system. We analyze a set of likely scenarios under which the system would be effective and determine how drivers would likely respond. Typical night crashes due to visibility involve pedestrians in unexpected locations, at moderately high speed limits (e.g., main arterials and rural roads). There does not seem to be a problem of drivers not looking at the road, but simply their inability to see pedestrians with their low beam headlights.

Exposure to alerts. The incidence of alerts is an important factor in choosing the alert characteristics. It is important to estimate the probability of true alerts, which would prevent crashes and near-misses. It is just as important to consider the probability of nuisance alerts with the currently available technology, which typically represent alerts in scenarios where drivers do not expect to respond because of their interpretation of the situation, or their comfortable risk level. As was shown in the safety analysis, true night vision alerts are very rare. As such, most alerts a driver would receive would be nuisance alerts. If nuisance alerts are also very few, the driver is unlikely to recognize a true alert on time because of unfamiliarity. If, on the other hand, nuisance alerts are many, they are likely to decrease user acceptance and reliance on the system. 
User acceptance and annoyance. Subjective preference is typically related to the obtrusiveness of the alert (in terms of timing, modality, and loudness) and the frequency with which it occurs (see discussion in Campbell, et al., 2007). They are also related to the user's understanding and ability to predict the meaning of the alert. In night vision systems, there is a misperception among drivers as to the true visibility limitations and the selective degradation of vision at night (Leibowitz and Owen, 1977). Despite our safety concerns, drivers may be more accepting of video systems that show them the road than systems that show them only pedestrians.

Possible distraction. Video displays with continuous information about the road may distract drivers from the primary task of looking at the road ahead. If a driver has to scan a night vision display continuously so as not to miss pedestrians and other objects of interest, they may be distracted from addressing other occasional needs of the driving task.

Expected behavior adaptation. Drivers may adapt their behavior to the presence of a night vision system by reducing scanning of complex displays and/or by taking greater risks (e.g., driving faster) at night. In contrast, a night vision system that would alert the driver of unexpected pedestrians might educate the driver of their visibility limitations and perhaps result in a positive adaptation of their driving patterns at night.

\section{IMPLICATIONS FOR THE DESIGN OF EFFECTIVE NIGHT VISION SYSTEMS}

There are several implications of the safety estimates and the additional considerations for the design of effective night vision systems. First, there is clearly a potential safety benefit from night vision systems that focus on the detection and avoidance of pedestrians, animals, and cyclists. Second, the rarity of events that an individual driver is likely to experience requires special consideration. Many of the considerations for the design of crash avoidance systems (e.g., Campbell et al., 2007) apply well in this context. For example, if warnings are not heard frequently, drivers may respond to them slowly, or not respond at all. To address this issue, it may be helpful to provide the driver with nonintrusive alerts to noncritical cases, which would facilitate learning of appropriate responses. The alerts would have to be nonintrusive so as not to distract the driver, but sufficiently noticeable to draw the driver's attention in rare situations.

The potential safety benefit of night vision systems has to be weighed against the cost of continuously displaying information that may not improve, and may even hinder, their safety and the safety of others. This cost may include driver distraction if drivers need to either look away from or draw their attention away from the road intermittently. Furthermore, it is possible that some drivers will use night vision systems only when they think they cannot see well enough, and ignore the system when they feel confident that they can see well. This phenomenon might be explained by the selective degradation theory (Leibowitz and Owens, 1977). Leibowitz and Owens proposed that at low levels of illumination, typical in night driving, certain "focal" visual capabilities (such as detecting pedestrians) are significantly impaired, whereas certain "ambient" visual capabilities (such as the visual guidance needed to steer the vehicle) are relatively well preserved. They suggested that drivers are not fully aware of this selective degradation.

Several night vision systems are currently offered in the automotive market. Their displays are installed in the central console, instrument cluster, or on a head-up display. They provide a good 
view of the road scene ahead and improve the driver's ability to detect pedestrians and animals (Hankey,, Keifer, and Gibbons, 2006). Some systems enhance the image as far as $300 \mathrm{~m}$ ahead (Kallhammer, 2006). Automatic pedestrian detection at shorter distances is already available, and is expected to be implemented in some of the next-generation night vision systems.

Systems without automatic pedestrian detection do not explicitly focus on pedestrians. Some provide better detection distances than others, but there is no indication to the driver about the importance of detecting pedestrians relative to other tasks. Because these systems provide information that is continuously available without special alerting, a driver might miss a rare event, especially if the expectation or level of vigilance is low. During an event, the driver might need to continuously scan the display to confirm the presence of a pedestrian instead of looking outside the vehicle and focusing on an avoidance maneuver. Systems with automatic pedestrian detection can highlight pedestrians, thus conveying their importance to the driver. They are expected to be effective for rare events because they draw the driver's attention whenever there is a pedestrian ahead. After a pedestrian has been detected, there is almost no need for the driver to look again at the display because the pedestrian is already highlighted. Interruption during the event itself is therefore expected to be minimal. A potential for negative impact exists because the display is regularly on and drivers may tend to look at it more often than necessary. Possible problems with automation may arise if the driver becomes overly reliant on the system's ability to detect pedestrians.

\section{A SIMPLE AND EFFECTIVE DISPLAY CONCEPT}

We propose that pedestrian detection can be improved with a simplified display that has the potential to comply with all the discussed safety criteria. The proposed system focuses on pedestrians and is designed to be effective for low-probability pedestrian events. During an event, there is no major interruption by the system. It is based on automatic pedestrian detection and is subject to some level of overreliance and complacency, but because it is not very intrusive, it can be designed with a greater bias towards false positives than false negatives (misses), thus reducing the effects of the overreliance problem.

The proposed simplified night vision system consists of a pedestrian icon to indicate the presence of a pedestrian near the future path of the vehicle. The icon is visible, but nonintrusive. It is designed so that it is easy to detect, especially at night, without directly looking at it. It alerts a driver to the presence of a pedestrian ahead, and is expected to increase detection distance. A likely driver response to the pedestrian icon is attending carefully and preparing to slow down. In unopposed situations, the driver may turn on the high-beam headlights. In some cases, such as when driving in heavily populated areas, the vehicle will already be slow, and the driver may already be fully aware of the presence of pedestrians. The system is primarily intended, however, for higher speed driving and roads on which pedestrians are less expected and pedestrian crashes are likely to occur because of darkness (Sullivan and Flannagan, 2001).

Another potential benefit of the proposed system is that it may facilitate learning. If the pedestrian icon turns on during the day and night every time there is a pedestrian, drivers will be able to learn about the performance of the system by confirming the alerts with what they see during daytime. Additionally, they are likely to receive implicit feedback about the visibility 
problem of pedestrian detection at night. Although the display concept is simple, the system would require an underlying set of sensors and computing algorithms that are not available on most current systems. It is expected, however, that in the next few years the hardware and software for such systems will be available, as research is currently underway in this direction.

\section{ON-THE-ROAD TESTING OF THE PROPOSED DESIGN}

Sixteen drivers, eight younger (ages 23-30, mean 26) and eight older (ages 62-76, mean 66), drove an instrumented vehicle with an experimenter in the back during regular nighttime traffic on a 20 -mile-route that consisted of rural roads. Seven pedestrians, simulated by inflatable dummies dressed in dark Jeans cloths, were positioned on the shoulder of the road along the route. Subjects drove the route twice and indicated verbally as soon as they saw a pedestrian. On one pass the pedestrians were all on the right side and on the other pass they were on either the right or the left side of the road. During half of the experiment the simple night vision display was active, lighting up as soon as the pedestrian was within $150 \mathrm{~m}$ in front of the vehicle (Figure 1). During the other half, it was off all the time. The order of conditions was balanced across subjects. Detection distances were analyzed using a mixed-model design. A detection distance of zero was assigned to cases in which there was no detection of the pedestrian. For more details see Tsimhoni, et al. (2007).

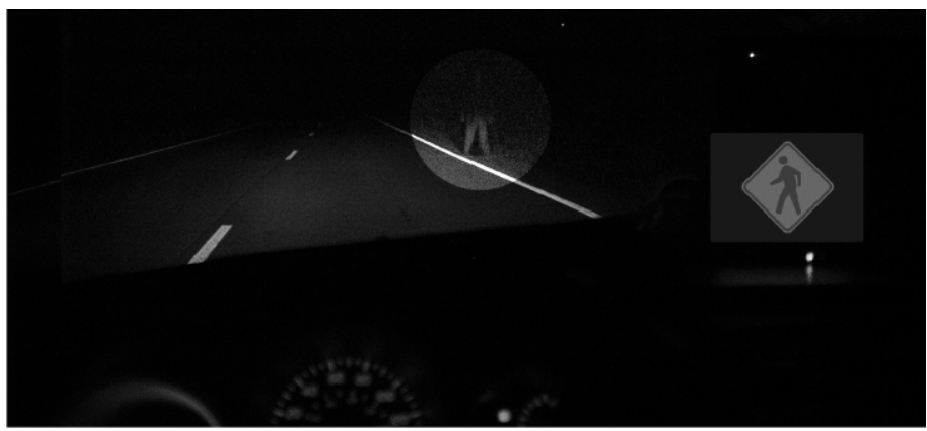

Figure 1. Driver's view of a pedestrian mannequin at a distance of about 30 m (The image has been modified to enhance the pedestrian and adjust the lighting of the pedestrian icon.)

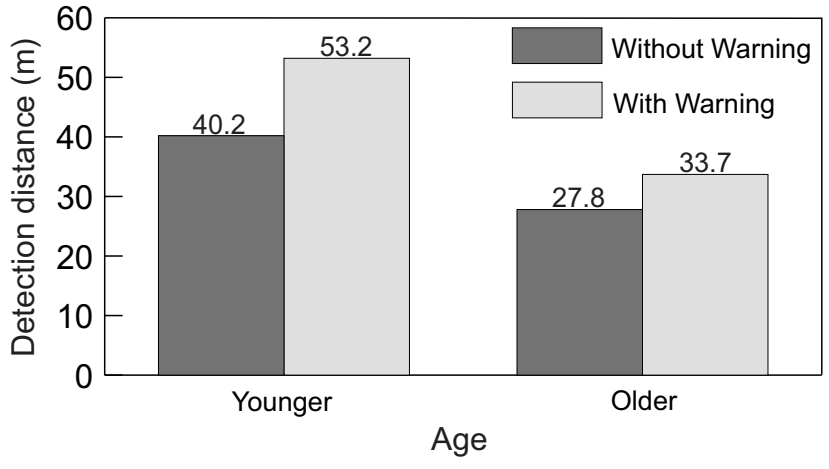

Figure 2. Pedestrian detection distance by age and warning

Detection distance without a warning $(34 \mathrm{~m})$ was significantly shorter than with a warning $(44 \mathrm{~m}) F(5,84)=2.92, p<0.05$. As expected, older subjects had shorter detection distances 
$(31 \mathrm{~m})$ than did younger subjects $(46 \mathrm{~m}) F(1,10.7)=6.22, p<0.05$. The interaction between age and warning was not statistically significant (Figure 2 ).

\section{DISCUSSION}

Detection distance of pedestrians in a naturalistic environment improved from 34 to $44 \mathrm{~m}$ with the pedestrian icon. The proportion of missed pedestrians decreased correspondingly from $13 \%$ to $5 \%$. The improvement is attributed to directing the driver's attention to the presence of a pedestrian. It is speculated that if the drivers were completely unexpecting of an event, the effect would be larger, as shown in the past by Roper and Howard (1938). In that study it was found that detection distance to a pedestrian that was entirely unexpected was half of when the driver was expecting it. We argue that the simple night vision display tested in this experiment has the potential to improve safety at night. Preventing pedestrian collisions due to darkness can potentially save up to 2,300 deaths per year in the U.S., although a single driver is not very likely to encounter such an event in their entire lifetime. An effective solution should not only improve the driver's visibility of the pedestrian, but also be as nonintrusive as possible. The proposed approach for displaying minimalistic information that is both simple and effective is unlikely to distract the driver as would more complex, often cluttered, displays. The operation of such a pedestrian icon during both the day and night is also likely to improve driver awareness to visibility limitations at night and to the problem of pedestrian visibility in general.

The current experiment may underestimate the real-world benefits of the warning provided by the icon in terms of how the warning might affect general preparation to respond to and avoid a pedestrian. The present experiment measured only the effects of the warning on seeing distance. In addition to improving seeing distance, the warning provided by the icon would allow drivers in the real world to begin some avoidance responses to pedestrians before they are visible-e.g., turn on high beam headlights, slow down, prepare to steer, or brake. The results of the present study are not sufficient in themselves to evaluate the overall effectiveness of the sort of night vision system that is described here, but they do demonstrate the potential effectiveness of the minimal driver interface for increasing pedestrian detection. It is likely that keeping the driver interface as simple as possible would also have favorable effects on the driver workload imposed by the system, although that aspect of the system was not directly tested here. Although we have proposed what is virtually the simplest possible driver interface, there are clearly other solutions between our proposed display and existing displays (e.g., Graf et al., 2005). For example, the display might include information about the distance to the detected pedestrian, the relative location of the pedestrian and the number of new pedestrians. More research is needed to understand what design would provide the most benefit. The decision on whether to add elements to the display should include an assessment of the added workload or distraction they would add when the display is not actively helping the driver avoid pedestrian crashes. Furthermore, there should be an assessment of whether including additional information in the display might provide benefits in terms of driver acceptance or understanding of the system.

Finally, the same method of analysis and conceptual system operation can be applied to other collision avoidance systems, where the information conveyed to the driver indicates the presence of other objects that are not easily seen by the driver, such as vehicles in adjacent lanes. 


\section{ACKNOWLEDGMENTS}

This research was supported by the University of Michigan Industry Affiliation Program for Human Factors in Transportation. Members include Alps Automotive/Alpine Electronics, Autoliv, Avery Dennison, Bendix, BMW, Bosch, Com-Corp Industries, DaimlerChrysler, Decoma Autosystems, Denso, Federal-Mogul, Ford, GE, General Motors, Gentex, Grote Industries, Hella, Honda, Ichikoh Industries, Koito Manufacturing, Lang-Mekra North America, Magna Donnelly, Muth, Nissan, North American Lighting, Northrop Grumman, OSRAM Sylvania, Philips Lighting, Renault, Sisecam, SL Corporation, Stanley Electric, Toyota Technical Center USA, Truck-Lite, Valeo, Visteon/ACH, and 3M Personal Safety Products. Information about the Affiliation Program is available at: http://www.umich.edu/ industry/

\section{REFERENCES}

Campbell, J.L., Richard, C.M., Brown, J.L., and McCallum, M. (2007). Crash Warning System Interfaces: Human Factors Insights and Lessons Learned (NHTSA Technical Report No. DOT HS 810 697). Washington, DC: National Highway Traffic Safety Administration.

Graf, T., Seifert, K., Meinecke, M., and Schmidt, R. (2005). Advanced Night Vision Systems Concepts. In, Proceedings of the $12^{\text {th }}$ world congress on Intelligent Transport Systems. Washington, DC: ITS-America.

Hankey, J.M., Kiefer, R.J., \& Gibbons, R.B. (2005). Quantifying the Pedestrian Detection Benefits of the General Motors Night Vision System. SAE Paper \#2005-01-0443. Warrendale, PA: Society of Automotive Engineers.

Kallhammer, J.-E. (2006). Night vision: Requirements and possible roadmap for FIR and NIR systems. In, Proceedings of SPIE Vol 6198, 61980F 1-11. Strasbourg, France.

Knapp, K.K., Kenert, C., and Witte, A. (2005). Statewide and upper Midwest summary of deervehicle crash and related data from 1993 to 2003 (Report No. DVCIC-03). Madison, WI: Midwest Regional University Transportation Center.

Leibowitz, H.W., and Owens, D.A. (1977). Nighttime driving accidents and selective visual degradation. Science 197, 422-423.

Roper, V.J., and Howard, E.A. (1938). Seeing with Motor Car Headlamps. Transactions of the Illuminating Engineering Society, 33(5), 417-438.

Rumar, K. (2003). Infrared night vision systems and driver needs (SAE Technical Paper Series No. 2003-01-0293). Warrendale, PA: Society of Automotive Engineers.

Sullivan, J.M., and Flannagan, M.J. (2001). Characteristics of pedestrian risk in darkness (Technical Report UMTRI-2001-33). Ann Arbor, MI: The University of Michigan Transportation Research Institute.

Tsimhoni, O., Flannagan, M.J., Mefford, M.L., and Takenobu, N. (2007). Improving pedestrian detection with a simple display for night vision systems (Technical Report No. UMTRI-20072). Ann Arbor, MI: The University of Michigan Transportation Research Institute. 